\title{
TITLE:
}

\section{Exact stability analysis of 2-D systems using LMIS}

AUTHOR(S):

Ebihara, Y; Ito, Y; Hagiwara, T

\section{CITATION:}

Ebihara, Y ... [et al]. Exact stability analysis of 2-D systems using LMIs. IEEE TRANSACTIONS ON AUTOMATIC CONTROL 2006, 51(9): 1509-1513

\section{ISSUE DATE:}

2006-09

URL:

http://hdl.handle.net/2433/39973

\section{RIGHT:}

(c)2006 IEEE. Personal use of this material is permitted. However, permission to reprint/republish this material for advertising or promotional purposes or for creating new collective works for resale or redistribution to servers or lists, or to reuse any copyrighted component of this work in other works must be obtained from the IEEE. 


\title{
Exact Stability Analysis of 2-D Systems Using LMIs
}

Yoshio Ebihara, Yoshimichi Ito, and Tomomichi Hagiwara

\begin{abstract}
In this note, we propose necessary and sufficient conditions for the asymptotic stability analysis of two-dimensional (2-D) systems in terms linear matrix inequalities (LMIs). By introducing a guardian map for the set of Schur stable complex matrices, we first reduce the stability analysis problems into nonsingularity analysis problems of parameter-dependent complex matrices. Then, by means of the discrete-time positive real lemma and the generalized $\mathcal{S}$-procedure, we derive LMI-based conditions that enable us to analyze the asymptotic stability in an exact (i.e., nonconservative) fashion. It turns out that, by employing the generalized $\mathcal{S}$-procedure, we can derive smaller size of LMIs so that the computational burden can be reduced.
\end{abstract}

Index Terms-Linear matrix inequalities (LMIs), stability analysis, twodimensional (2-D) systems.

\section{INTRODUCTION}

In this note, we address asymptotic stability analysis problems of two-dimensional (2-D) systems described by the Fornasini-Marchesini second model [6]

$$
\begin{array}{r}
x(i+1, j+1)=A_{1} x(i, j+1)+A_{2} x(i+1, j) \\
A_{1}, A_{2} \in \mathbf{C}^{n \times n} .
\end{array}
$$

Precise definition of the asymptotic stability of the 2-D system was first made in [5]. Since then, various types of necessary and sufficient conditions have been proposed for the analysis of the asymptotic stability. We summarize some of them in the following proposition.

Proposition 1: [6] The following conditions are equivalent.

i) The 2-D system (1) is asymptotically stable.

ii) $\operatorname{det}\left(I_{n}-z_{1} A_{1}-z_{2} A_{2}\right) \neq 0$ for all $\left(z_{1}, z_{2}\right) \in \overline{\mathbf{D}} \times \overline{\mathbf{D}}$ where $\overline{\mathbf{D}}$ denotes the closure of the open unit disc $\mathbf{D}$ on the complex plane.

iii) $\rho(A(\theta))<1$ for all $\theta \in[0,2 \pi]$ where $A(\theta):=A_{1}+e^{j \theta} A_{2}$ and $\rho(\cdot)$ denotes the spectral radius.

Unfortunately, the conditions in ii) and iii) are not numerically tractable since they should be checked at infinitely many points over

Manuscript received April 3, 2004; revised March 22, 2005 and March 23, 2006. Recommended by Associate Editor D. Nesic. This work was supported in part by the Ministry of Education, Culture, Sports, Science, and Technology of Japan under Grant-in-Aid for Young Scientists (B) 15760314.

Y. Ebihara and T. Hagiwara are with the Department of Electrical Engineering, Kyoto University, Kyoto 615-8510, Japan (e-mail: ebihara@kuee.kyoto-u.ac.jp).

Y. Ito is with the Graduate School of Engineering, Osaka University, Osaka 565-0871, Japan.

Digital Object Identifier 10.1109/TAC.2006.880789 
the range of $\left(z_{1}, z_{2}\right)$ or $\theta$. As clearly shown in the condition ii), the stability analysis problems of 2-D systems are regarded to be "two repeated scalar block problems" in the framework of $\mu$-analysis [13] and their difficulties are now widely recognized. To get around these difficulties, Galkowski et al. [4] showed the effectiveness of linear matrix inequalities (LMIs) [2], [3] and opened up a new horizon for the analysis and synthesis of 2-D systems. In particular, they provided a concise linear matrix inequality (LMI) condition for condition ii) to hold, which can be used also for state-feedback stabilizing controller synthesis [4]. Exactly the same LMI condition was provided also by Ooba [11]. As we will clarify later on, however, this LMI condition is nothing but the one resulting from D-scaling on two repeated scalar block $\mu$-problems and hence it does not allow us to achieve exact stability analysis [15]. On the other hand, Ito et al. [7] made another effort to convert condition iii) into feasibility tests of LMIs via Lyapunov's stability theory. However, these approaches employ Lyapunov functions of restricted form [7] and thus satisfactory results have not been obtained on the exactness of the resulting LMI-based conditions.

In stark contrast with these existing results, in this note, we propose necessary and sufficient conditions for the asymptotic stability analysis of 2-D systems in terms of LMIs. To achieve this, we first introduce a guardian map [1] for the set of Schur stable complex matrices and reduce the stability analysis condition iii) given in Proposition 1 into nonsingularity analysis of parameter-dependent complex matrices. Then, by means of the discrete-time positive real lemma [14] and the generalized $\mathcal{S}$-procedure [9], [10], we readily derive parameter-independent LMIs that enable us to analyze the asymptotic stability in an exact fashion. It turns out that, by working with the generalized $\mathcal{S}$-procedure, we can derive smaller size of LMIs so that the computational burden can be reduced. Through numerical experiments, we illustrate the effectiveness of the proposed LMI conditions and examine its computational complexity.

We use the following notations in this note. For a matrix $A \in \mathbf{C}^{n \times n}$, its transpose, complex conjugate and complex conjugate transpose are denoted by $A^{T}, \bar{A}$ and $A^{*}$, respectively. For matrices $A$ and $B$, we denote by $A \otimes B$ their Kronecker product. For a matrix $A \in \mathbf{C}^{n \times m}$ with $\operatorname{rank}(A)=r<n, A^{\perp} \in \mathbf{C}^{(n-r) \times n}$ is a matrix such that $A^{\perp} A=0$ and $A^{\perp}\left(A^{\perp}\right)^{*}>0$. The symbol $\mathbf{H}_{n}$ denote the sets of $n \times n$ Hermitian matrices.

\section{GUARDian Map fOR StABILITy ANALYSIS OF 2-D SYSTEMS}

The notion of guardian map [1] plays a key role in this note. In this section, we first review its definition and introduce a guardian map for the set of Schur stable complex matrices.

Definition 1 (Guardian Map [1]): Let $\nu: \mathbf{C}^{n \times n} \rightarrow \mathbf{R}$ be a given map. Then $\nu$ is said to be an guardian map for an open set $\mathcal{M} \subset \mathbf{C}^{n \times n}$ if $\nu(M) \neq 0$ for $M \in \mathcal{M}$ and $\nu(M)=0$ for $M \in \partial \mathcal{M}$ where $\partial \mathcal{M}$ denotes the boundary of $\mathcal{M}$.

Lemma 1 (Guardian Map for the Set of Schur Stable Matrices [1]): Let us consider the map $\nu: \mathbf{C}^{n \times n} \rightarrow \mathbf{R}$ described by

$$
\nu(M)=\operatorname{det}\left(\bar{M} \otimes M-I_{n} \otimes I_{n}\right) .
$$

Then, the map $\nu(\cdot)$ is a guardian map for the set of Schur stable $n \times n$ complex matrices.

The usefulness of guardian maps is well-known when we deal with robust $\mathcal{D}$-stability analysis problems of uncertain parameter-dependent matrices [1]. Following the discussions in [1], we can obtain the next lemma.
Lemma 2: Let us consider the 2-D system described by (1). Then the system is asymptotically stable if and only if the following two conditions hold:

i) $\rho\left(A_{1}+A_{2}\right)<1$;

ii) $\operatorname{det}(\mathcal{A}(\theta)) \neq 0$ for all $\theta \in[0,2 \pi]$ where

$$
\begin{aligned}
\mathcal{A}(\theta): & =\bar{A}(\theta) \otimes A(\theta)-I_{n} \otimes I_{n} \\
& =\mathcal{A}_{-1} e^{-j \theta}+\mathcal{A}_{0}+\mathcal{A}_{1} e^{j \theta} \\
\mathcal{A}_{0} & :=\bar{A}_{1} \otimes A_{1}+\bar{A}_{2} \otimes A_{2}-I_{n} \otimes I_{n} \\
\mathcal{A}_{-1} & :=\bar{A}_{2} \otimes A_{1} \\
\mathcal{A}_{1}: & =\bar{A}_{1} \otimes A_{2} .
\end{aligned}
$$

Proof: We first note that $\operatorname{det}(\mathcal{A}(\theta))=\nu(A(\theta))$ at each fixed $\theta \in$ $[0,2 \pi]$. Hence, from the condition iii) in Proposition 1 and Definition 1 , the necessity of i) and ii) is obvious. To prove the sufficiency, suppose that the condition i) holds, i.e., $\rho(A(\theta))<1$ at $\theta=0$. Then, from the continuity of the eigenvalues of $A(\theta)$ with respect to $\theta$, and from the properties of the guardian map stated in Definition 1, the condition ii) ensures $\rho(A(\theta))<1$ for all $\theta \in[0,2 \pi]$. This completes the proof.

In Lemma 2, we have reduced the asymptotic stability analysis problem of the 2-D system (1) into nonsingularity analysis problem of the parameter-dependent complex matrix $\mathcal{A}(\theta)$. However, it is still hard to check the nonsingularity of $\mathcal{A}(\theta)$ at infinitely many points over the range $[0,2 \pi]$. To overcome this difficulty, in the next section, we further reduce the nonsingularity analysis problem into feasibility tests of parameter-independent LMIs.

\section{EXACT STABILITY ANALYSIS USING LMIS}

\section{A. Reduction to LMI via the Positive Real Lemma}

In this subsection, we reduce the condition ii) in Lemma 2 into feasibility tests of a parameter-independent LMI. To this end, we follow the results by Ohara and Sasaki [12], which enable us to reduce parameter-dependent LMIs into parameter-independent ones by applying the discrete-time positive real lemma [14] in a particular way.

At each fixed $\theta \in[0,2 \pi]$, we see that $\operatorname{det}(\mathcal{A}(\theta)) \neq 0$ holds if and only if $\mathcal{A}(\theta)^{*} \mathcal{A}(\theta)>0$. From (3), the latter condition is also equivalent to $\mathcal{G}(\theta)+\mathcal{G}(\theta)^{*}>0$ where

$$
\begin{aligned}
\mathcal{G}(\theta) & :=\mathcal{G}_{0}+\mathcal{G}_{-1} e^{-j \theta}+\mathcal{G}_{-2} e^{-2 j \theta} \\
\mathcal{G}_{0} & :=\frac{1}{2}\left(\mathcal{A}_{-1}^{*} \mathcal{A}_{-1}+\mathcal{A}_{0}^{*} \mathcal{A}_{0}+\mathcal{A}_{1}^{*} \mathcal{A}_{1}\right) \\
\mathcal{G}_{-1} & :=\mathcal{A}_{0}^{*} \mathcal{A}_{-1}+\mathcal{A}_{1}^{*} \mathcal{A}_{0} \\
\mathcal{G}_{-2} & :=\mathcal{A}_{1}^{*} \mathcal{A}_{-1} .
\end{aligned}
$$

In relation to the trigonometric polynomial $\mathcal{G}(\theta)$, let us follow the methodology in [12] and construct a discrete-time system $G(z)$ of the form

$$
\begin{aligned}
G(z): & =\left[\begin{array}{ll}
A_{G} & B_{G} \\
\hline C_{G} & D_{G}
\end{array}\right] \quad\left[\begin{array}{ll}
A_{G} & B_{G} \\
C_{G} & D_{G}
\end{array}\right]: \\
& =\left[\begin{array}{ccc}
0 & I_{n^{2}} & 0 \\
0 & 0 & I_{n^{2}} \\
\mathcal{G}_{-2} & \mathcal{G}_{-1} & \mathcal{G}_{0}
\end{array}\right] .
\end{aligned}
$$

Then, we see that $\mathcal{G}(\theta)+\mathcal{G}(\theta)^{*}>0$ holds for all $\theta \in[0,2 \pi]$ if and only if $G(z)$ is strictly positive real, i.e., $G\left(e^{j \theta}\right)+G\left(e^{j \theta}\right)^{*}>0(\forall \theta \in$ $[0,2 \pi])$. It is well-known that the positive realness of discrete-time systems can be verified via single (i.e., parameter-independent) LMI by 
means of the positive real lemma [14]. Consequently, we readily obtain the following theorem that provides a necessary and sufficient condition for the asymptotic stability of the 2-D system (1) in terms of LMIs.

Theorem 1: The 2-D system (1) is asymptotically stable if and only if the following two conditions hold.

i) $\rho\left(A_{1}+A_{2}\right)<1$.

ii) There exist $Q_{11}, Q_{22} \in \mathbf{H}_{n^{2}}$ and $Q_{12} \in \mathbf{C}^{n^{2} \times n^{2}}$ such that

$$
\left[\begin{array}{ccc}
0 & 0 & \mathcal{G}_{-2}^{*} \\
0 & 0 & \mathcal{G}_{-1}^{*} \\
\mathcal{G}_{-2} & \mathcal{G}_{-1} & \mathcal{G}_{0}+\mathcal{G}_{0}^{*}
\end{array}\right]>\left[\begin{array}{ccc}
Q_{11} & Q_{12} & 0 \\
Q_{12}^{*} & Q_{22}-Q_{11} & -Q_{12} \\
0 & -Q_{12}^{*} & -Q_{22}
\end{array}\right]
$$

where $\mathcal{G}_{0}, \mathcal{G}_{-1}$ and $\mathcal{G}_{-2}$ are defined by (6) and (4).

If the matrices $A_{1}$ and $A_{2}$ are real, we can restrict $Q_{11}, Q_{22}$, and $Q_{12}$ in (8) to be real.

Proof: From the positive real lemma [14], the system $G(z)$ given in (7) is strictly positive real if and only if there exists $Q \in \mathbf{H}_{2 n^{2}}$ such that

$$
\begin{aligned}
& {\left[\begin{array}{cc}
0 & C_{G}^{*} \\
C_{G} & D_{G}+D_{G}^{*}
\end{array}\right]} \\
& \quad>\left[\begin{array}{cc}
A_{G} & B_{G} \\
I_{2 n^{2}} & 0
\end{array}\right]^{*}\left[\begin{array}{cc}
-Q & 0 \\
0 & Q
\end{array}\right]\left[\begin{array}{cc}
A_{G} & B_{G} \\
I_{2 n^{2}} & 0
\end{array}\right] .
\end{aligned}
$$

If $A_{1}$ and $A_{2}$ are real, the matrices $A_{G}, B_{G}, C_{G}$ and $D_{G}$ are all real and thus we can restrict $Q$ to be real [8]. By partitioning $Q$ in (9) as

$$
\begin{aligned}
Q & =\left[\begin{array}{ll}
Q_{11} & Q_{12} \\
Q_{12}^{*} & Q_{22}
\end{array}\right] \\
Q_{11}, Q_{22} & \in \mathbf{H}_{n 2} \\
Q_{12} & \in \mathbf{C}^{n^{2} \times n^{2}}
\end{aligned}
$$

and substitute the matrices $A_{G}, B_{G}, C_{G}$ and $D_{G}$ given in (7), we see that the inequality condition (9) reduces to (8). This completes the proof.

Now, we have derived an LMI-based condition for the asymptotic stability analysis of the 2-D system (1). It should be emphasized that, in stark contrast with the existing results [4], [7], [11], the condition in Theorem 1 is surely nonconservative. Nevertheless, it leaves room for improvement from the viewpoint of numerical computation. The size of LMI in (8) is $3 n^{2}$ and the number of complex scalar variables are $n^{2}\left(2 n^{2}+1\right)$. These unfortunately prevent us from dealing with large size problems. To reduce computational burden, in the next subsection, we derive smaller size of LMI by the generalized $\mathcal{S}$-procedure [9], [10].

\section{B. Reduction to LMI via Generalized S-Procedure}

The generalized $\mathcal{S}$-procedure, introduced by Iwasaki et al. [9], [10], has proved to be very useful for robustness analysis and synthesis of control systems. Roughly speaking, by working with the generalized $\mathcal{S}$-procedure, we can reduce parameter-dependent LMIs into parameter-independent ones without any conservatism. The results in [9] and [10] are now reviewed.

Lemma 3 (Generalized $\mathcal{S}$-Procedure [9], [10]): Let a matrix $\Theta \in$ $\mathbf{H}_{n}$ and a subset $\mathcal{S} \subset \mathbf{H}_{n}$ be given. Suppose $\mathcal{S}$ is lossless [8][9]. Then, the following conditions are equivalent.

i) $\zeta^{*} \Theta \zeta>0 \quad \forall \zeta \in \mathcal{H} \quad \mathcal{H}:=\left\{\zeta \in \mathbf{C}^{n}: \zeta \neq 0 \quad \zeta^{*} S \zeta \geq\right.$ $0 \quad \forall S \in \mathcal{S}\}$.

ii) There exists $S \in \mathcal{S}$ such that $\Theta>S$.
Precise definition of the lossless set can be found in [9]. In the following lemma, we show how the nonsingularity analysis problem of the parameter-dependent matrix $\mathcal{A}(\theta)$ can be reduced into a feasibility test of parameter-independent LMIs via the generalized $\mathcal{S}$-procedure.

Lemma 4: Let us consider the 2-D system described by (1). Define $\mathcal{A}(\theta)$ and $\mathcal{A}_{i}(i=-1,0,1)$ by (3) and (4), respectively. We further define $\mathcal{W}:=\left[\begin{array}{lll}\mathcal{A}_{-1} & \mathcal{A}_{0} & \mathcal{A}_{1}\end{array}\right]$. Then, the following conditions are equivalent.

i) $\mathcal{A}(\theta)^{*} \mathcal{A}(\theta)>0 \quad \forall \theta \in[0,2 \pi]$.

ii)

$$
\begin{aligned}
& \zeta^{*} \mathcal{W}^{*} \mathcal{W} \zeta>0 \quad \forall \zeta \in \mathcal{K} \\
& \mathcal{K}:=\left\{\zeta=\left[\begin{array}{lll}
\zeta_{2}^{*} & \zeta_{1}^{*} & \zeta_{0}^{*}
\end{array}\right]^{*} \in \mathbf{C}^{3 n^{2}}: \zeta \neq 0,\right. \\
& \left.\quad \exists s \in \partial \mathbf{D} \text { such that }\left[\zeta_{2}^{*} \zeta_{1}^{*}\right]^{*}=s\left[\zeta_{1}^{*} \zeta_{0}^{*}\right]^{*}\right\} .
\end{aligned}
$$

iii)

$$
\begin{aligned}
& \zeta^{*} \mathcal{W}^{*} \mathcal{W} \zeta>0 \quad \forall \zeta \in \mathcal{L} \\
& \mathcal{L}:=\left\{\zeta \in \mathbf{C}^{3 n^{2}}: \zeta \neq 0 \quad \zeta^{*} S \zeta \geq 0 \quad \forall S \in \mathcal{S}_{\mathbf{D}}\right\} \\
& \mathcal{S}_{\mathbf{D}}:=\left\{\Gamma^{T}\left[\begin{array}{cc}
Q & 0 \\
0 & -Q
\end{array}\right] \Gamma: Q \in \mathbf{H}_{2 n^{2}}\right\} \\
& \Gamma:=\left[\begin{array}{cccc}
I_{n^{2}} & 0 & 0 & 0 \\
0 & I_{n^{2}} & I_{n^{2}} & 0 \\
0 & 0 & 0 & I_{n^{2}}
\end{array}\right] .
\end{aligned}
$$

iv) There exists $Q \in \mathbf{H}_{2 n^{2}}$ such that

$$
\mathcal{W}^{*} \mathcal{W}>\Gamma^{T}\left[\begin{array}{cc}
Q & 0 \\
0 & -Q
\end{array}\right] \Gamma
$$

v) There exists $Q \in \mathbf{H}_{2 n^{2}}$ such that

$$
\left(\mathcal{W}^{*}\right)^{\perp}\left(\Gamma^{T}\left[\begin{array}{cc}
Q & 0 \\
0 & -Q
\end{array}\right] \Gamma\right)\left(\mathcal{W}^{*}\right)^{\perp^{*}}<0 .
$$

If the matrix $\mathcal{W}$ is real, the equivalence still holds when we restrict $Q$ in iii)-v) to be real.

Proof: The equivalence of the conditions i) and ii) is obvious. Hence, we prove only the equivalence of ii)-v).

Equivalence of ii) and iii): To prove the equivalence, it is enough to show that $\mathcal{K}=\mathcal{L}$. Suppose $\zeta=\left[\zeta_{2}^{*} \zeta_{1}^{*} \zeta_{0}^{*}\right]^{*} \in \mathcal{K}$. Then, we see from the definition of $\mathcal{K}$ that the following equality holds for all $Q \in \mathbf{H}_{2 n^{2}}$.

$$
\zeta^{*}\left(\Gamma^{T}\left[\begin{array}{cc}
Q & 0 \\
0 & -Q
\end{array}\right] \Gamma\right) \zeta=\left(s^{*} s-1\right)\left[\begin{array}{l}
\zeta_{1} \\
\zeta_{0}
\end{array}\right]^{*} Q\left[\begin{array}{l}
\zeta_{1} \\
\zeta_{0}
\end{array}\right]=0
$$

This shows that $\zeta \in \mathcal{L}$ and, hence, $\mathcal{K} \subset \mathcal{L}$ holds. On the other hand, suppose $\zeta=\left[\begin{array}{lll}\zeta_{2}^{*} & \zeta_{1}^{*} & \zeta_{0}^{*}\end{array}\right]^{*} \in \mathcal{L}$. Then, we have from the definition of $\mathcal{L}$ that

$$
\begin{aligned}
& \left(\left(\left[\begin{array}{l}
\zeta_{2} \\
\zeta_{1}
\end{array}\right]\left[\begin{array}{l}
\zeta_{2} \\
\zeta_{1}
\end{array}\right]^{*}-\left[\begin{array}{l}
\zeta_{1} \\
\zeta_{0}
\end{array}\right]\left[\begin{array}{l}
\zeta_{1} \\
\zeta_{0}
\end{array}\right]^{*}\right) Q\right) \geq 0 \\
& \forall Q \in \mathbf{H}_{2 n^{2}}
\end{aligned}
$$

It can be shown that the previous condition holds if and only if

$$
\left[\begin{array}{l}
\zeta_{2} \\
\zeta_{1}
\end{array}\right]\left[\begin{array}{l}
\zeta_{2} \\
\zeta_{1}
\end{array}\right]^{*}-\left[\begin{array}{l}
\zeta_{1} \\
\zeta_{0}
\end{array}\right]\left[\begin{array}{l}
\zeta_{1} \\
\zeta_{0}
\end{array}\right]^{*}=0
$$


which implies $\left[\zeta_{2}^{*} \zeta_{1}^{*}\right]^{*}=s\left[\zeta_{1}^{*} \zeta_{0}^{*}\right]^{*}$ for some $s \in \partial \mathbf{D}$ [14]. This clearly shows that $\zeta \in \mathcal{K}$ and thus $\mathcal{L} \subset \mathcal{K}$. To summarize, we can conclude that $\mathcal{K}=\mathcal{L}$

Equivalence of iii) and iv): From [8], the set $\mathcal{S}_{\mathrm{D}}$ is lossless. Hence, the equivalence of iii) and iv) follows from the generalized $\mathcal{S}$-procedure stated in Lemma 3.

Equivalence of iv) and v): This is a direct consequence of Finsler's theorem [2].

By noting that the real case results can be shown by simply taking the real part of the matrix variable $Q \in \mathbf{H}_{2 n^{2}}$, we complete the proof.

In the above proof, the equivalence of $\mathrm{i}$ ) and iv) can also be shown by [7, Th. 1], where an LMI-based method for checking the positive definiteness of finite-order Fourier series is presented.

We are now in a position to state the following theorem.

Theorem 2: The 2-D system (1) is asymptotically stable if and only if the following two conditions hold.

i) $\rho\left(A_{1}+A_{2}\right)<1$.

ii) There exist $Q_{11}, Q_{22} \in \mathbf{H}_{n}$, and $Q_{12} \in \mathbf{C}^{n^{2} \times n^{2}}$ such that

$$
\left[\begin{array}{c}
\mathcal{A}_{-1}^{*} \\
\mathcal{A}_{0}^{*} \\
\mathcal{A}_{1}^{*}
\end{array}\right]^{\perp}\left[\begin{array}{ccc}
Q_{11} & Q_{12} & 0 \\
Q_{12}^{*} & Q_{22}-Q_{11} & -Q_{12} \\
0 & -Q_{12}^{*} & -Q_{22}
\end{array}\right]\left[\begin{array}{c}
\mathcal{A}_{-1}^{*} \\
\mathcal{A}_{0}^{*} \\
\mathcal{A}_{1}^{*}
\end{array}\right]^{\perp^{*}}<0
$$

where $\mathcal{A}_{-1}, \mathcal{A}_{0}$ and $\mathcal{A}_{1}$ are defined by (14).

If the matrices $A_{1}$ and $A_{2}$ are real, we can restrict $Q_{11}, Q_{22}$ and $Q_{12}$ in (14) to be real.

Proof: The assertions follow immediately from Lemmas 2 and 4.

When comparing (8) and (14), we see that the number of scalar variables is the same. However, the size of LMI has been reduced form $3 n^{2}$ to $3 n^{2}-r$ where $r=\operatorname{rank}\left(\left[\mathcal{A}_{-1} \mathcal{A}_{0} \mathcal{A}_{1}\right]^{*}\right)$. In particular, if the matrix $\mathcal{A}_{1}$ or $\mathcal{A}_{0}$ is nonsingular, the size of LMI (14) becomes $2 n^{2}$ and the LMI (14) can be written more concisely by using explicit expressions of $\left(\left[\begin{array}{lll}\mathcal{A}_{-1} & \mathcal{A}_{0} & \mathcal{A}_{1}\end{array}\right]^{*}\right)^{\perp}$. This is summarized in the next corollary.

Corollary 1: Let us consider the 2-D system (1) and define the matrices $\mathcal{A}_{i}(i=-1,0,1)$ by (4). If $\mathcal{A}_{1}$ is nonsingular, then the 2-D system (1) is asymptotically stable if and only if the condition i) in Theorem 2 and the following condition holds.

i a)There exist $X_{11}, X_{22} \in \mathbf{H}_{n^{2}}$ and $X_{12} \in \mathbf{C}^{n^{2} \times n^{2}}$ such that

$$
\begin{array}{r}
{\left[\begin{array}{cc}
-\mathcal{A}_{1} & 0 \\
0 & -\mathcal{A}_{1} \\
\mathcal{A}_{-1} & \mathcal{A}_{0}
\end{array}\right]^{*}\left[\begin{array}{ccc}
X_{11} & X_{12} & 0 \\
X_{12}^{*} & X_{22}-X_{11} & -X_{12} \\
0 & -X_{12}^{*} & -X_{22}
\end{array}\right]} \\
\times\left[\begin{array}{cc}
-\mathcal{A}_{1} & 0 \\
0 & -\mathcal{A}_{1} \\
\mathcal{A}_{-1} & \mathcal{A}_{0}
\end{array}\right]<0 .
\end{array}
$$

On the other hand, if $\mathcal{A}_{0}$ is nonsingular, then the 2-D system (1) is asymptotically stable if and only if the condition i) in Theorem 2 and the following condition hold:

ii b)There exist $Y_{11}, Y_{22} \in \mathbf{H}_{n^{2}}$ and $Y_{12} \in \mathbf{C}^{n^{2} \times n^{2}}$ such that

$$
\begin{array}{r}
{\left[\begin{array}{cc}
-\mathcal{A}_{0} & 0 \\
\mathcal{A}_{-1} & \mathcal{A}_{1} \\
0 & -\mathcal{A}_{0}
\end{array}\right]^{*}\left[\begin{array}{ccc}
Y_{11} & Y_{12} & 0 \\
Y_{12}^{*} & Y_{22}-Y_{11} & -Y_{12} \\
0 & -Y_{12}^{*} & -Y_{22}
\end{array}\right]} \\
\\
\times\left[\begin{array}{cc}
-\mathcal{A}_{0} & 0 \\
\mathcal{A}_{-1} & \mathcal{A}_{1} \\
0 & -\mathcal{A}_{0}
\end{array}\right]<0 .
\end{array}
$$

Proof: If $\mathcal{A}_{1}$ is nonsingular, we have

$$
\left[\begin{array}{c}
\mathcal{A}_{-1}^{*} \\
\mathcal{A}_{0}^{*} \\
\mathcal{A}_{1}^{*}
\end{array}\right]^{\perp}=\left[\begin{array}{cc}
-\mathcal{A}_{1} & 0 \\
0 & -\mathcal{A}_{1} \\
\mathcal{A}_{-1} & \mathcal{A}_{0}
\end{array}\right]^{*}\left[\begin{array}{ccc}
\mathcal{A}_{1}^{-1} & 0 & 0 \\
0 & \mathcal{A}_{1}^{-1} & 0 \\
0 & 0 & \mathcal{A}_{1}^{-1}
\end{array}\right]^{*}
$$

Hence, by defining $X_{11}:=\left(\mathcal{A}_{1}^{-1}\right)^{*} Q_{11} \mathcal{A}_{1}^{-1}, X_{22} \quad:=$ $\left(\mathcal{A}_{1}^{-1}\right)^{*} Q_{22} \mathcal{A}_{1}^{-1}$ and $X_{12}:=\left(\mathcal{A}_{1}^{-1}\right)^{*} Q_{12} \mathcal{A}_{1}^{-1}$, the condition (15) readily follows from (14). By similar arguments, we can derive (16) from (14) when $\mathcal{A}_{0}$ is nonsingular.

Let us now give brief comments on the equivalence of the conditions in Theorems 1 and 2. Since both conditions are necessary and sufficient, the condition (8) should be able to be rewritten in the form of (14). This can be seen easily once we have (11) in Lemma 4 . Indeed, by letting $Q_{11}, Q_{22}$ and $Q_{12}$ in (8) as $Q_{11}:=-\mathcal{A}_{-1}^{*} \mathcal{A}_{-1}+\hat{Q}_{11}, Q_{22}:=$ $-\left(\mathcal{A}_{-1}^{*} \mathcal{A}_{-1}+\mathcal{A}_{0}^{*} \mathcal{A}_{0}\right)+\hat{Q}_{22}$ and $Q_{12}:=-\mathcal{A}_{-1}^{*} \mathcal{A}_{0}+\hat{Q}_{12}$, the condition (8) can be rewritten equivalently as follows.

$$
\left[\begin{array}{c}
\mathcal{A}_{-1}^{*} \\
\mathcal{A}_{0}^{*} \\
\mathcal{A}_{1}^{*}
\end{array}\right]\left[\begin{array}{c}
\mathcal{A}_{-1}^{*} \\
\mathcal{A}_{0}^{*} \\
\mathcal{A}_{1}^{*}
\end{array}\right]^{*}>\left[\begin{array}{ccc}
\hat{Q}_{11} & \hat{Q}_{12} & 0 \\
\hat{Q}_{12}^{*} & \hat{Q}_{22}-\hat{Q}_{11} & -\hat{Q}_{12} \\
0 & -\hat{Q}_{12}^{*} & -\hat{Q}_{22}
\end{array}\right]
$$

Hence, the condition (14) also follows from (8). However, the reformulation into (17) shown above cannot be seen naturally from (8). Thus, the discussions based on the generalized $\mathcal{S}$-procedure is indispensable for the reduction of the size of LMIs.

Remark 1: For the asymptotic stability analysis of the 2-D system (1), we can readily obtain an LMI-based sufficient condition by D-scaling [15]. To see this, let us note that the condition ii) of Proposition 1 can be rewritten, equivalently as

$$
\begin{aligned}
\operatorname{det}\left(I_{2 n}-\left[\begin{array}{cc}
z_{1} I_{n} & 0 \\
0 & z_{2} I_{n}
\end{array}\right]\left[\begin{array}{c}
I_{n} \\
I_{n}
\end{array}\right]\left[\begin{array}{ll}
A_{1} & A_{2}
\end{array}\right]\right) \\
\\
\quad \neq 0 \text { for all }\left(z_{1}, z_{2}\right) \in \overline{\mathbf{D}} \times \overline{\mathbf{D}}
\end{aligned}
$$

Hence, by applying D-scaling with the scaling matrix

$$
W=\left[\begin{array}{cc}
W_{1}^{\frac{1}{2}} & 0 \\
0 & W_{2}^{\frac{1}{2}}
\end{array}\right] W_{1}>0 \quad W_{2}>0 \quad W_{1}, W_{2} \in \mathbf{C}^{n \times n}
$$

we see that the 2-D system (1) is asymptotically stable if the following LMI condition holds:

$$
\begin{aligned}
& {\left[\begin{array}{cc}
A_{1}^{*}\left(W_{1}+W_{2}\right) A_{1}-W_{1} & A_{1}^{*}\left(W_{1}+W_{2}\right) A_{2} \\
A_{2}^{*}\left(W_{1}+W_{2}\right) A_{1} & A_{2}^{*}\left(W_{1}+W_{2}\right) A_{2}-W_{2}
\end{array}\right]<0} \\
& W_{1}>0 \quad W_{2}>0 \text {. }
\end{aligned}
$$

It should be noted that this LMI condition was derived by Galkowski et al. [4] and Ooba [11] without applying D-scaling techniques directly. In particular, it was shown in [4] that the LMI condition (20) is promising for state-feedback controller design, whereas the analysis conditions in this note are not necessarily suitable for controller synthesis.

Note however that the LMI condition (20) is only sufficient and far from necessity [15]. Even though the proposed conditions (8) and (14) should be computationally more demanding than (20), they enable us 
TABLE I

COMPUTATION RESULTS $\left(\left(\gamma_{l 0}, \gamma_{u 0}\right)=(0.8720,1.5975)\right)$

\begin{tabular}{|c|c|c|c|}
\hline Methods & $\hat{\gamma}$ & Iterations in bisection & CPU time [s] \\
\hline The LMI condition (16) & 0.9978 & 14 & 32.66 \\
\hline The LMI condition (20) [4], [11] & 1.0393 & 14 & 2.26 \\
\hline \hline Lower bound by a discrete search & 0.9978 & - & - \\
\hline
\end{tabular}

to achieve exact stability analysis. We illustrate these points through numerical experiments in the next section.

\section{ILLUSTRATIVE EXAMPLES}

Let us consider the 2-D system described by (1) where

$$
\begin{aligned}
A_{1} & =\left[\begin{array}{cccc}
0.5 & 0.5 & 0.4 & 1.1 \\
0.1 & -0.1 & 0.6 & 0.1 \\
0 & 0 & 0 & 0 \\
0 & 0 & 0 & 0
\end{array}\right] \\
A_{2} & =\left[\begin{array}{cccc}
0 & 0 & 0 & 0 \\
0 & 0 & 0 & 0 \\
-0.1 & -0.1 & -0.2 & -0.5 \\
-0.2 & 0.6 & -0.1 & -0.7
\end{array}\right] .
\end{aligned}
$$

Our problem here is to find a value $\gamma$ defined by

$$
\gamma=\inf \left\{\bar{\gamma} \in \mathbf{R}: \rho\left(A_{1}+e^{j \theta} A_{2}\right)<\bar{\gamma} \quad \forall \theta \in[0,2 \pi]\right\} .
$$

To this end, we apply the analysis conditions discussed in the preceding section and seek for the estimate $\hat{\gamma}$ of $\gamma$ by a bisection search. For the fast convergence of the bisection algorithms, we first need to compute strict lower bounds and upper bounds of $\gamma$. Concerning the lower bounds, it is apparent that $\gamma_{l 0} \leq \gamma$ where $\gamma_{l 0}:=\rho\left(A_{1}+A_{2}\right)$. On the other hand, we see that $\gamma \leq \gamma_{u 0}$ holds where

$$
\gamma_{u 0}:=\|T\|_{\infty} \quad T(z):=\left[\begin{array}{l|l}
0 & A_{2} \\
\hline I & A_{1}
\end{array}\right] .
$$

Taking these $\gamma_{l 0}$ and $\gamma_{u 0}$ as initial values for the lower bound $\gamma_{l}$ and the upper bound $\gamma_{u}$, we carry out a bisection search under the stopping criterion $\gamma_{u}-\gamma_{l}<10^{-4}$. For comparison, we examine the following two stability analysis conditions.

1 The LMI condition (14) in Theorem 2. In particular, since the matrix $\mathcal{A}_{0}$ turns out to be nonsingular in this problem, we adopt (16) to achieve fast computation.

2 The LMI condition (20) provided in [4], [11], which can be obtained also by D-scaling.

The computation results are shown in Table I. ${ }^{1}$ To examine the exactness of the analysis conditions, we also performed a discrete search of $\rho\left(A_{1}+e^{j \theta} A_{2}\right)$ over $[0,2 \pi]$ with griding points 100000 and obtain a lower bound of $\gamma$ as shown in Table I. From the results in Table I, we can confirm that the proposed condition yields exact value for $\gamma$, even though it is computationally more demanding than (20).

\section{CONCLUSION}

In this note, we proposed LMI-based conditions for the asymptotic stability analysis of 2-D systems. The proposed conditions are

${ }^{1}$ All LMI-related computations are carried out with MATLAB and SeDuMI105, on Pentium IV $3.6 \mathrm{GHz}$. promising in the sense that they are nonconservative and enable us to achieve exact stability analysis. Unfortunately, however, the analysis conditions are computationally demanding since the size and the number of scalar variables of the proposed LMIs grow rapidly with respect to the problem size. We showed that the generalized $\mathcal{S}$-procedure is effective to reduce the size of LMIs but the computational complexity could be further reduced by exploring different guardian maps from the one employed in this note. On the other hand, even though we have concentrated our attention on LMI-based analysis conditions in this note, it should be also interesting to explore exact analysis methods by means of simple and efficient algebraic computations. These are subjects of our further investigation in the future.

\section{ACKNOWLEDGMENT}

The authors would like to thank the anonymous reviewers for their careful reading and valuable suggestions.

\section{REFERENCES}

[1] B. R. Barmish, New Tools for Robustness of Linear Systems. New York: Macmillan, 1994.

[2] S. P. Boyd, L. E. Ghaoui, E. Feron, and V. Balakrishnan, Linear Matrix Inequalities in System and Control Theory. Philadelphia, PA: SIAM, 1998.

[3] P. Gahinet, A. Nemirovskii, A. J. Laub, and M. Chilali, LMI Control Toolbox. Natick, MA: The Mathworks, Inc., 1994.

[4] K. Galkowski, E. Rogers, S. Xu, J. Lam, and D. H. Owens, "LMIs-A fundamental tool in analysis and controller design for discrete linear repetitive processes," IEEE Trans. Circuits Syst. I, Fundam. Theory Appl., vol. 49, no. 6, pp. 768-778, Jun. 2002.

[5] E. Fornasini and G. Marchesini, "Doubly-indexed dynamical systems: State-Space models and structural properties," Math. Syst. Theory, vol. 12, pp. 59-72, 1978

[6] E. Fornasini and G. Marchesini, "Stability analysis of 2-D systems," IEEE Trans. Circuits Syst., vol. CAS-27, no. 12, pp. 1210-1217, Dec. 1980.

[7] Y. Ito, K. Hayashi, and H. Fujiwara, "Stability analysis and $H_{\text {inf }}$ norm computation of 2-D discrete systems using linear matrix inequalities," in Proc. IEEE Conf. Decision Control, Las Vegas, NV, 2002, pp. 3306-3311.

[8] T. Iwasaki, LMI and Control. Tokyo, Japan: Shokodo, 1997.

[9] T. Iwasaki, G. Meinsma, and M. Fu, "Generalized $\mathcal{S}$-procedure and finite frequency KYP lemma," Math. Prob. Eng., vol. 6, pp. 305-320, 2000.

[10] T. Iwasaki and S. Hara, "Generalized KYP lemma: Unified frequency domain inequalities with design applications," IEEE Trans. Autom. Control, vol. 50, no. 1, pp. 41-59, Jan. 2005.

[11] T. Ooba, "On stability analysis of 2-D systems based on 2-D lyapunov matrix inequalities," IEEE Trans. Circuits Syst. I, Fundam. Theory Appl., vol. 47, no. 8, pp. 1263-1265, Aug. 2000.

[12] A. Ohara and Y. Sasaki, "On solvability and numerical solutions of parameter-dependent differential matrix inequality," in Proc. IEEE Conf. Decision Control, Orlando, FL, 2001, pp. 3593-3594.

[13] A. Packard and J. C. Doyle, "The complex structured singular value," Automatica, vol. 29, no. 1, pp. 71-109, 1993.

[14] A. Rantzer, "On the Kalman-Yakubovič-Popov lemma," Syst. Control Lett., vol. 28, pp. 7-10, 1996.

[15] K. Zhou and J. C. Doyle, Essentials of Robust Control. Upper Saddle River, NJ: Prentice-Hall, 1998. 